\title{
LEGAL REGULATION OF INTERNATIONALIZATION IN RUSSIAN PUBLIC INSTITUTES OF HIGHER EDUCATION: A CASE OF VOLGOGRAD INSTITUTE OF MANAGEMENT
}

\author{
Ekaterina V. Stepanova \\ Volgograd Institute of Management - Branch of the Russian Presidential Academy \\ of National Economy and Public Administration, Volgograd, Russian Federation \\ Natalia E. Buletova \\ Volgograd Institute of Management - Branch of the Russian Presidential Academy \\ of National Economy and Public Administration, Volgograd, Russian Federation
}

Introduction: Russian Public higher education system is dynamically changing due to gradual integration into the global educational market. The authors trace the adaptation of public higher educational institutions to modern conditions, study a real case of the Volgograd Institute of Management, branch of RANEPA specializing in public policy and business administration, its trends and issues in the internationalization process related to legal status of a branch of a public institution; suggest a series of measures to optimize its academic mobility trends. The purpose of the study is to determine the actual trends of the international activities of government funded organizations taking into account the current trends in the public reform of higher education in Russia and limitations arising from its legal status of a branch. Techniques: such methods as case study, system, comparative legal and logical analyses were applied. Results: it is proved that the success of internationalization process in Russian higher education in academic, research and consulting areas largely depends on the factors of regional level, legal status of public institutions, available resources as well as on branch network structure. Conclusions: it was found that in order to overcome limitations in the process of internationalization government-funded educational institutions with a status of a branch should focus on internal internalization id est on creation of interdisciplinary pilot educational programs with foreign partners within the framework of short-term and medium-term academic mobility on the basis of individual international academic trajectories and the creation of courses in English and the program "Russian as a Foreign Language".

Key words: legal status of branch, higher education, internationalization, public institutes, academic mobility.

\section{ПРАВОВОЕ РЕГУЛИРОВАНИЕ МЕЖДУНАРОДНОЙ ДЕЯТЕЛЬНОСТИ РОССИЙСКИХ БЮДЖЕТНЫХ ОРГАНИЗАЦИЙ ВЫСШЕГО ОБРАЗОВАНИЯ: СИТУАЦИОННЫЙ АНАЛИЗ ВИУ РАНХИГС}

\author{
Екатерина Владимировна Степанова \\ Волгоградский институт управления - филиал Российской академии народного хозяйства \\ и государственной службы при Президенте РФ, г. Волгоград, Российская Федерация

\section{Наталья Евгеньевна Булетова} \\ Волгоградский институт управления - филиал Российской академии народного хозяйства \\ и государственной службы при Президенте РФ, г. Волгоград, Российская Федерация
}


Введение: система высшего образования в России динамично развивается, совершенствуется в условиях продолжающейся глобализации рынка образовательных услуг, интеграции в Болонский процесс, диктующий четкие нормативно-правовые и методологические основы национальной модели высшего образования. Российские бюджетные образовательные учреждения высшего образования сталкиваются с серьезными проблемами, которые связаны как с вопросами достаточности, доступности финансовых ресурсов, но и с недостаточным опытом и проблемами адаптации зарубежной модели бакалавриата и магистратуры на фоне отказа от традиционного пятилетнего специалитета. Представлены результаты адаптации бюджетных высших учебных заведений к условиям, нормам и ценностям интернационализации современного рынка образования, а также сложности и проблемы, связанные с правовым статусом «филиала». Цель статьи - выявить и охарактеризовать актуальные тенденции в международной деятельности бюджетных образовательных учреждений с учетом содержания государственной реформы высшего образования в Российской Федерации. Применяемые методы включают в себя кейсисследование, методы системного анализа, сравнительный, правовой и логический методы. Результаты исследования свидетельствуют о высоком уровне зависимости процесса интернационализации российских высших учебных заведений в академических, исследовательских и консультационных областях от региональных факторов и диспропорциях социального, экономического и правового поля, от правового статуса государственных учреждений и юридического лица, имеющихся ресурсов и статуса филиала. Вывод: во время интернационализации для преодоления существующих правовых и ресурсных ограничений в развитии бюджетных организаций высшего образования, имеющих статус филиала на уровне субъекта федерации рекомендуется укреплять внутреннюю интернализацию, создавая: междисциплинарные пилотные образовательные программы совместно с зарубежными партнерами в рамках краткосрочной и среднесрочной академической мобильности преподавателей и студентов по индивидуальным международным академическим траекториям; разработка курсов на английском языке и программ «русский язык как иностранный» для повышения привлекательности предлагаемых образовательных программ для иностранных студентов.

Ключевые слова: правовой статус филиала, высшее образование, интернационализация, бюджетные организации, академическая мобильность.

INSERM 37.014

BCB 67.400

\title{
REGLEMENTATION JURIDIQUE DE L'ACTIVITE INTERNATIONALE DES ORGANISATIONS BUDGETAIRES RUSSES DE L'ENSEIGNEMENT SUPERIEUR: ANALYSE SITUATIONNELLE DE L'INSTITUT DE GESTION DE VOLGOGRAD
}

\author{
Ekaterina V. Stepanova \\ Institut de l'Administration Publique de Volgograd - filiale de l'Académie Russe \\ de l'Economie Nationale et de l'Administration Publique, Volgograd, Russie

\section{Natalya E. Buletova} \\ Institut de l'Administration Publique de Volgograd - filiale de l'Académie Russe \\ de l'Economie Nationale et de l'Administration Publique, Volgograd, Russie
}

La Introduction. L'enseignement supérieur public russe change de manière dynamique en raison de son intégration progressive sur le marché mondial éducatif en particulier dans le Processus de Bologne. Les universités publics russes font face à des défis importants qui ne résument pas seulement à des difficultés de finances, mais

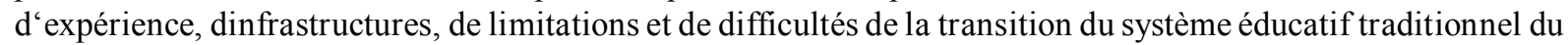
licence de spécialistes de cinq ans au système de baccalauréat et de maîtrise, etc. Les universités publics russes ont fait le choix dêtre présentes sur la scène internationale en sintégrant au système denseignement supérieur. L'auteur de larticle retrace l'adaptation des établissements d'enseignement supérieur publics aux conditions dans le contexte actuel, aux tendances et aux problèmes liés au nouveau rôle et au statut juridique, aux nouvelles normes et valeurs. Le but de l'article est de déterminer les tendances actuelles de l'activité internationale des 
etablissements publics en tenant compte des tendances actuelles de la réforme publique de l'enseignement supérieur dans la Fédération de Russie. Les techniques appliquées incluent les méthodes danalyse systémique, la méthode juridique comparative et la méthode logique. Les résultats: Il est prouvé que le succès du processus d'internationalisation de l'établissement denseignement supérieur russe dans les domaines académiques, de recherche et de consultation dépend largement des facteurs régionaux, du statut juridique des institutions publiques, des ressources disponibles ainsi que de la structure du réseau de succursales/filiales. La conclusion: lors de l'internationalisation pour surmonter les limitations des institutions d'enseignement financées par le gouvernement avec un statut de branche, il est recommender de créer des programme interdisciplinaires sur la base des trajectoires académiques internationales individuelles; introduire les cours en anglais et le programme "russe comme langue étrangère".

Mots-clés: statut juridique de la branche, enseignement supérieur, internationalisation, organisations budgétaires, mobilité académique.

\section{La présentation du cadre et du contexte}

L'Institut de l'administration publique de Volgograd, filiale de 1'Académie Russe d'Économie Nationale et d'Administration Publique compte 3428 étudiants de premier cycle et de maîtrise $(5,23 \%$ du contingent des sujets de la Fédération de Russie) dont 26,0\% sont financé par l'état russe et $74,0 \%$ pays pour les services éducatifs.

La coopération internationale est théoriquement attribuée aux domaines prioritaires du développement; à l'aide de méthodes quantitatives et qualitatives des activités partenariat nous avons analysé l'état actuel des choses. Les activités internationales se concentrent, selon les tendances d'internationalisation des universités russes, sont organisées à un niveau suffisant, toutefois, les indicateurs d'efficacité sont positifs dans certains domaines, mais ont une tendance négative dans les autres.

L'Institut de l'administration publique de Volgograd comprennent la réputation dans la communauté internationale de l'enseignement supérieur, un réseau bien développé de partenaires (par rapport aux autres filiales) qui comprend environ 20 établissements d'enseignement et les organisations internationales de différents pays (1); La disponibilité de la formation internationale et des stages pour les étudiants et les enseignants (5); le nombre d'étudiants d'autres universités qui ont participé aux programmes à court terme (4); la courte mobilité sortante, la participation à des événements internationaux des représentants de L'Institut (3), l'organisation d'événements internationaux sur la base de L'Institut (2), la participation aux échanges internationals des jeunes (Fig. 1).

\section{Les domaines positifs du développement de la coopération internationale de}

Les indicateurs négatifs des activités internationales incluent un nombre insuffisants d'étudiants étrangers (à l'exclusion des pays de la CEI) qui ont terminé leurs études dans les programmes de premier cycle, des programmes spécialisés de 5 ans, ses programmes de maîtrise, de troisième cycle (1-7), ainsi que le nombre de professeurs et des chercheurs étrangers travaillant dans l'institut au moins 1 semestre (8-9), le montant des fonds reçus des activités R \& D des citoyens étrangers et personnes morales étrangers.

Les indicateurs de suivi de l'efficacité des activités internationales de l'Institut, faites par le ministère de l'Éducation et de la Science de la Fédération de Russie sont présentés dans la Fig. 2.

Il est à noter que l'information analytique sur l'efficacité des activités internationales du ministère de l'Éducation et de la Science prennent en compte unnombre limité d'indicateurs de l'activité universitaire, et sont limités par les chiffres de la mobilité académique des étudiants et des professeurs à long terme (au moins 6 mois), par le nombre d'étudiants étrangers inscrits dans un cycle complet d'éducation. Le développement de ces branches d'activités internationales est compliqué dans l'Institut de l'Administration Publique de Volgograd, filiale del'Académie Russe d'Économie Nationale et d'Administration Publique, d'une part, en raison du profil de l'université, qui comprend les sciences de la société (Pour les grands groupes de domaines : sciences psychologiques, l'économie et la gestion, la sociologie et le travail social, le droit, les sciences politiques et études régionales, linguistique et études littéraires) avec des caractéristiques régionales importantes, qui ne sont pas attrayantes pour les étudiants étrangers. 


\section{$-\mathrm{O}-$}

Valeurs médianes des indicateurs d'efficacité des filiales publiques et municipales

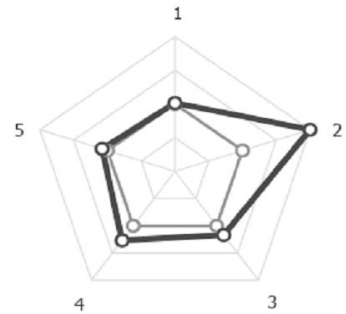

Indicateurs d'efficacité de la filiale (l'Institut de l'Administration publique de Volgograd, filale de l'Académie Russe d'Economie Nationale et d'Administration Publique)

de l'Administration publique de Volgograd, filale de l'Académie Russe d'Economie Nationale et d'Administration Publique)
Valeurs médianes des indicateurs d'efficacité des filiales publiques et municipales dans la région de Volgograd

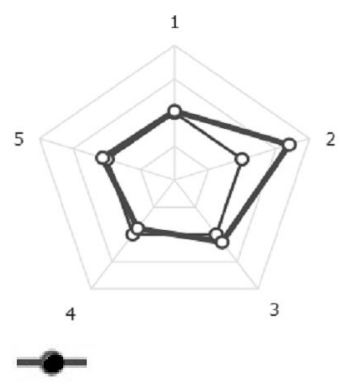

Indicateurs d'efficacité de la filiale (l'Institut de l'Administration publique de Volgograd, filale de l'Académie Russe d'Economie Nationale et d'Administration Publique) de l'Administration publique de Volgograd

Fig. 1. Indicateurs de suivi de l'efficacité des activités internationales de l'Institut de l'Administration Publique de Volgograd, filiale de l'Académie Russe d'Économie Nationale et d'Administration Publique (Information analytique sur les résultats du suivi du fonctionnement du réseau de l'Académie russe des sciences au premier trimestre 2017)

Source: Documents d'information et d'analyse sur les résultats du suivi de l'efficacité des sections de l'Académie russe des sciences au premier trimestre de 2017. 1 C Enterprise (accès 11/11/2017).

Note d'applicationles les données sont structurées par l'auteur pour plus de clarté.

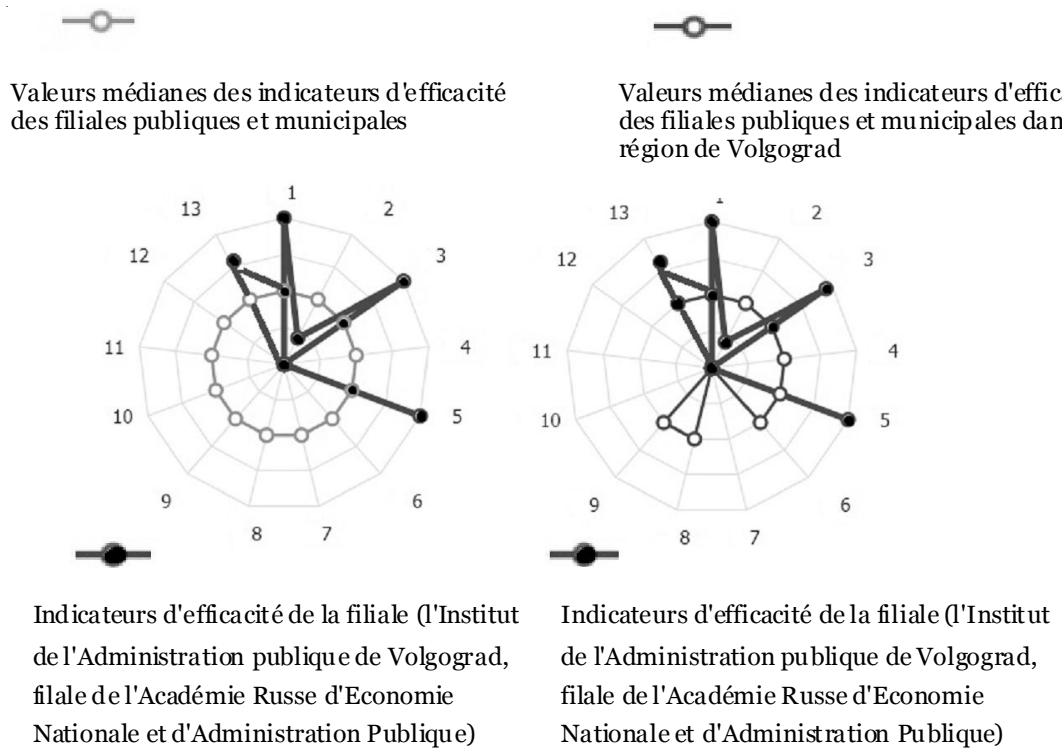

Fig. 2. Informations analytiques sur l'efficacité des activités internationales dans l'Institut de l'Administration Publique de Volgograd, filiale de l'Académie Russe d'Économie Nationale et d'Administration Publique en 2017

Source: Documents d'information et d'analyse sur les résultats du suivi de l'efficacité des établissements d'enseignement supérieur en 2017. Disponible: http://indicators.miccedu.ru/monitoring/_vpo/inst.php?id=10001849 (accès 11/11/2017) [1].

Note d'applicationles les données sont structurées par l'auteur pour plus de clarté. 
En second lieu, il n'a pas de cours enseignés en anglais, ce qui contribuerait à accroître le nombre d'étudiants étrangers. Troisièmement, il n'y a pas de cours préparatoires "Russe comme langue étrangère" à l'université. En outre, la possibilité de la $\mathrm{R} \& \mathrm{D}$ pour les citoyens étrangers et les personnes morales étrangères dans l'Institut de l'Administration Publique de Volgograd sont également limitées en raison du statut de filiale et de l'incapacité de conclure des contrats directs avec des contreparties étrangères.

\section{L'analyse de la démarche scientifique: \\ les stratégies de l'internationalisation dans les instituts publics russes d'enseignement supérieur}

Selon la définition adoptée dans les études de l'OCDE, l'internationalisation de l'enseignement supérieur aux niveaux national, régional et institutionnel est généralement considérée comme un processus dans lequel les objectifs, les fonctions et d'enseignement supérieur acquièrent une dimension internationale [2]. Le concept d'internationalisation dans le domaine de l'enseignement supérieur dans la pratique internationale comporte traditionnellement deux aspects: l'internalisation intérieure et extérieure. L'internationalisation de l'éducation comprend de telles formes de coopération internationale comme la mobilité individuelle: mobilité des étudiants ou des professeurs à des fins éducatives; la mobilité des programmes éducatifs et la mobilité institutionnelle; la formation de nouvelles normes internationales de programmes éducatifs; l'intégration dans les curriculums de la dimension et des normes éducatives internationales; partenariat institutionnel, la création d'alliances stratégiques dans le domaine de l'éducation.

Selon la classification de l'OCDE, dans 1'Institut de l'Administration Publique de Volgograd, filiale de l'Académie Russe d'Économie Nationale et d'Administration Publique nous avons dégagé quatre stratégies de l'internationalisation (intérieure et extérieure) de l'enseignement supérieur qui sont appliquées avec des degrés variables de succès. Premièrement la stratégie de coordination (Mutual understanding approach) qui s'appuie sur les objectifs de long terme dans les domaines culturels et académiques et est réalisée par le soutien à la mobilité des étudiants et des professeures universitaires à travers des programmes d'échanges académiques, de partenariats institutionnels dans l'enseignement supérieur. Dans l'Institutde l'Administration Publique de Volgograd ce type de coopération est confirmée par les accords en vigueur et est effectuée avec les 12 universités parmi lesquelles l'Université d'Ostrava, l'Université d'économie de Bratislava, le Comité pour la jeunesse, les enfants et les affaires familiales sous l'administration de Cologne.

Deuxièmement, l'Institut de l'Administration Publique de Volgograd applique la stratégie pour attirer la main-d'œuvre qualifiée (Skilled migration approach) visée à attirer des spécialistes étrangers et des étudiants talentueux dans l'institut et dans la région de Volgograd pour assurer le développement économique et accroître la compétitivité dans l'économie du savoir [3]. Des spécialistes étrangers hautement qualifiés sont régulièrement invités à l'Institut (au moins 4 par an). Par exemple, une série de tables rondes dans le cadre de la conférence internationale "Problèmes réels de protection de l'environnement en Russie et aux Etats-Unis" avec la participation d'éminents experts dans le domaine du droit environnemental de la Fédération de Russie et du directeur de l'approvisionnement en eau département de la Direction de la protection civile de l'Agence fédérale de l'environnement du gouvernement des États-Unis.

Troisièmement, la stratégie génératrice de revenus (Revenue-generating approach) qui vise à générer du profit, repose sur les deux stratégies décrites ci-dessus. Dans son cadre, les services éducatifs sont entièrement payés par les étudiants étrangers et les subventions ne sont pas fournies [4]. En même temps, le coût de la formation pour les étudiants étrangers est plusieurs fois supérieur à celui des étudiants russes ce qui pourraient donner un revenu supplémentaire. Cependant, la principale filiale de l'Académie Russe d'Économie Nationale et d 'Administration Publique à Moscou n'accorde pas une autonomie significative aux succursales, de sorte que l'Institut de l'Administration Publique de Volgograd est limité dans la détermination de la politique financière et du coût de ses services éducatifs.

Quatrièmement, par la stratégie de renforcement des capacités (Capacity building approach) l'Institutde l'Administration Publique 
de Volgograd encourage parmi ses étudiants l'enseignement supérieur du cycle complet ou les programmes à court ou moyen terme dans des instituts partenariats étrangers à l'aide de tels que programmes que le soutien de la mobilité des employés de l'Etat, des professeurs, des scientifiques et des étudiants. Par exemple, il y a des échanges éducatifs et scientifiques avec l'Académie des Arts et des Sciences Angelico Constantiniana (Italie).

\section{La Discussion critique}

À la suite de la recherche, les résultats suivants ont été obtenus sur les aspects théoriques et pratiques de la mise en œuvre du processus de l'internationalisation des etablissements publics de la formation professionnelle supérieure, en cas particulier de l'Institut de l'administration publique de Volgograd filiale de l'Académie Russe d'Économie Nationale et d'Administration Publique.

Tenant compte des résultats du changement dans le statut juridique qui a influencé les capacités et les pouvoirs des établissements d'enseignement supérieur publiques dans le système d'enseignement international, les auteurs ont proposé une définition de celles-ci en tant que les exécutrices des services de la formation publics et municipaux et les participants membres des affaires économiques extérieures:

l'établissement public est une entité sans but lucratif agissant dans le domaine des services éducatifs, sociaux et culturels ayant un statut bivalent qui se manifeste dans l'obligation d'exécuter des services publics et municipaux y compris dans le domaine du partenariat avec les établissements internationaux, et en même temps dans la nécessité de déterminer les stratégies et les priorités de la coopération internationale réglementée par la politique économique extérieure, par la législation dans des conditions financières, structurelles et juridiques limitées.

Il existe des paradoxes du processus de 1'internationalisation des etablissements publics russes. Premièrement, l'état encourage la mise en œuvre des programmes de coopération internationale par les etablissements publics en offrant un certain nombre de prélèvements fiscaux, et introduit le critère d'exécution des programmes internationaux comme un des indicateurs de performance et d'efficacité de l'établissement, mais en même temps l'état limite les pouvoirs et les activités des etablissements publics (y compris, dans les domaines de partenariat académique et scientifique). Deuxièmement, la position intermédiaire de l'établissement public russe entre le système budgétaire d'état russe et l'environnement des affaires conduit à la complication des activités internationales.

Sur la base de la systématisation des résultats positifs et négatifs de la réforme des établissements publics, les auteurs ont dégagé des avantages et des inconvénients dans le domaine de l'internationalisation. Les avantages incluent le statut des exécutrices des services de la formation publics et municipaux ce qui crée une image de stabilité et donne un avantage concurrentiel dans la recherche de partenariat académique; le fait que les indicateurs des activités internationales font part de la performance lors de son évaluation; le développement de l'autonomie fonctionnelle et financière dans la formation et la disposition des ressources de l'établissement public, bien que les organisations autonomes aient l'indépendance de la prise de décision en raison de certaines conditions inhérentes à leur statut [5].

Les défauts de l'internationalisation dans les établissements publics comprennent le renforcement de la dépendance de l'efficacité des activités internationales sur le marché mondial de la politique internationale, du subventionnement et des caractéristiques des services offerts; perte du statut de "bénéficiaire des fonds budgétaires", cela a creusé le fossé entre les établissements publics et le système budgétaire dans son ensemble, renforçant la dualité de la nature et de la position, du statut d' établissements publics dans le domaine de la coopération internationale.

Considérant des problèmes rencontrés par tous les participants au processus de la coopération internationale dans les etablissements publics de la formation professionnelle supérieure russe (les organisations publiques subventionnées par le budget d'état russe, le gouvernement russe, les gouvernements étrangers, les partenaires internationaux, les consommateurs des services de formation), les auteurs ont définie les directions du développement des activités internationales des etablissements publics (en cas de 1'Institut de l'Administration publique de Volgograd, filiale de 
l'Académie Russe d'Économie Nationale et d'Administration Publique) visant à la croissance des avantages concurrentiels sur le marché des services éducatifs, les vecteurs du développement de l'ordre de l'interaction internationale des etablissements publics, et les ont différenciés selon les domaines de la coopération internationale.

Pour construire un modèle plus efficace de l'internationalisation des institutions publiques de la formation supérieure avec un statut de filiale, les auteurs ont proposé une série des mesures combinées de la coopération internationale (dans les domaines des activités scientifiques, de la mobilité académique, de formation professionnelle et complémentaire, ainsi que des analyses, de consultations et des expertises).

\section{Les résultats}

Les auteurs révèlent le contenu et la spécificité de l'activité internationale de l'établissement public dans le domaine de l'enseignement supérieur dans l'aspect régional (l'exemple de 1'Institut de l'Administration publique de Volgograd). Il est prouvé que pour surmonter les limitations des institutions d'enseignement financées par le gouvernement avec un statut de branche dans le processus d'internationalisation, il faut se concentrer sur la création des programmes de recherche interdisciplinaires sur la base des trajectoires académiques internationales individuelles et la création des formations supérieures enseignées en anglais et du programme "russe comme langue étrangère".

Pour déterminer la stratégie la plus prometteuse de l' internationalisation d'un établissement public de l'enseignement supérieur, on a évalue sélectivement l'environnement interne et externe de l'établissement public, ses indicateurs clé de performance internationale. L'efficacité des programmes internationaux de coopération existants avec les partenaires étrangers, la performance des activités de coopération internationale ont été estimées sur la base des résultats des rapports annuels publiés par l'institut. On a fait des recommandations pour le développement de l' internationalisation en particulier pour l'optimisation de la mobilité académique internationale des étudiants et des professeurs de l'Institut au niveau régional. Les propositions sont présentées selon le statut de l'institution, la structure verticale et horizontale de la coopération internationale, et la présence du réseau des filiales.

Conformément à l'objectif stratégique de la réforme du système éducatif russe et de l'augmentation de sa compétitivité dans l'espace international, conformément au concept du développement de long terme socioéconomique de la Fédération de Russie jusqu'en 2020 et de l'internationalisation en vertu de la législation en vigueur et la pratique d'autres établissements publics sur le marché des services d'enseignement fédéral et régional, nous estimons qu'il est nécessaire de recommander:

- stimuler la coopération scientifique internationale;

- réaliser le potentiel de la coopération académique (tous les cycles d'enseignement y compris des études de troisième cycle et de doctorat);

- développer des services d'expertise et des consultations selon le profil de l'institut (l'Administration publique, économie, le droit); - activer 1'internationalisation dans la formation professionnelle complémentaire.

\section{La Conclusion}

Pour surmonter les limitations du statue de filiale lors du processus d'internationalisation, il a été constaté que les établissements d'enseignement subventionnés par les pouvoirs publics devraient se concentrer sur l'internalisation intérieure, c'est-àdire sur la création des programmes éducatifs pilotes interdisciplinaires avec des partenaires étrangers dans le cadre de la mobilité académique à court et à moyen terme à la base des trajectoires académiques individuelles; lancer des cours en anglais et développer et mettre en œuvre un cours de formation programme "Le Russe comme langue étrangère".

Considérons des mesures d'optimisation de l'internalisation académique intérieure proposées selon la structure, le statut juridique, des conditions et les résultats escomptés. En proposant de renforcer la coopération académique internationale, nous sommes guidés par l'indicatrice cible de compétitivité internationale prévue par le Programme fédéral pour le développement de l'enseignement supérieur en 2016-2020 et nous proposons les orientations suivantes, qui 
contribueront également à l'amélioration des indicateurs de performance:

1. Le développement et la mise en œuvre de cours individuels, modules de programmes éducatifs, ainsi que des programmes éducatifs complets, tout ou en partie en langues étrangères, dans toutes les facultés de l'Institut en 2018-2020. Il est conseillé d'intégrer des modules en langues étrangères dans les domaines de formation les plus compétitifs.

2. La création des programmes éducatifs pilotes interdisciplinaires à cour terme en coopération avec des partenaires étrangers en 2018-2020.

3. Le développement et la mise en œuvre des programmes de double diplôme dans la période 2020-2023. La disponibilité de programmes de double diplôme contribuera à une augmentation des performances de la branche et des classement internationaux.

4. Améliorer la mobilité académique des professeurs et des étudiants dans le cadre d'un stage à court et moyen terme d'une durée d'une semaine à trois mois) Et d'une formation de longue durée au programme Erasmus + et avec des partenaires.

5. La construction des trajectoires académiques internationales individuelles et de programmes de mobilité académique. La coordination et la conclusion des accords de coopération dans le cadre d'Erasmus + et des programmes d'échange sur les accords de partenariat.

6. Créer un programme efficace pour l'étude du russe comme langue étrangère pour les citoyens étrangers dans le département préparatoire en 2018-2020.

7. L'invitation des professeurs et des spécialistes étrangers travaillant sous contrat à durée déterminée en 2018-2023. L'augmentation du nombre des professeurs étrangers dans les visites de courte durée et les conférences.

8. Augmenter le nombre des conférences ouvertes des spécialistes étrangers dans divers domaines de la connaissance sur le profil de l'université, des représentants des autorités publiques des pays étrangers dans les facultés dans le cadre de forums et de conférences scientifiques.

9. L'accréditation internationale des programmes éducatifs, la certification des modules et domaines de formation destinés aux citoyens étrangers.

10. Les stages d'enseignants et d'étudiants à travers des stages dans des organisations partenaires à des fins de formation continue, d'échanges académiques et culturels, de mobilité scientifique, de conférences et de développement de programmes d'études. L'augmentation du nombre d'enseignants ayant de l'expérience, des conférences et des stages dans des universités étrangères.

\section{REFERENCES}

1. Buletova N.E., Zlochevsky I.A., Sharkevich I.V., Stepanova E.V. Structural Analysis and Criteria for Assessment of State Economic Policy. International Journal of Applied Engineering Research, 2017, vol. 12, no. 17, pp. 6603-6614.

2. Information and Analytical Materials on the Results of Monitoring the Effectiveness of Higher Education Institutions in 2017. URL: http://indicators. miccedu.ru/monitoring/_vpo/inst.php?id=10001849 (accessed November 11, 2017). (in Russian)

3. Internationalization and Trade in Higher Education Opportunities and Challenges. OECD, 2004. $317 \mathrm{p}$.

4. Stepanova E.V. Òranslation studies: didactic training model of intercultural communication experts in higher education institutions of Russia. EDAMBA 2015 International Scientific Conference for Doctoral Students and Post-Doctoral Scholars: The Era of Science Diplomacy: Implications for Economics, Business. Bratislava, 2015, pp. 831-838.

5. Zadorina M.A. The quality of secondary vocational education as a guarantee of the implementation of the constitutional right to secondary vocational education. Pravovaya Paradigma [Legal Concept], 2017, no. 2 (16), pp. 71-76. DOI: https:// doi.org/10.15688/lc.jvolsu.2017.2.10. (in Russian). 


\section{Information about the Authors}

Ekaterina V. Stepanova, Candidate of Philological Sciences, Associate Professor, Department of Linguistics and Intercultural Communication, Volgograd Institute of Management - Branch of the Russian Presidential Academy of National Economy and Public Administration, Gagarina St., 8, 400131 Volgograd, Russian Federation, stepanova.volg@mail.ru.

Natalia E. Buletova, Candidate of Economic Sciences, Associate Professor, Department of Public Administration and Political Science, Volgograd Institute of Management - Branch of the Russian Presidential Academy of National Economy and Public Administration, Gagarina St., 8, 400131 Volgograd, Russian Federation, buletovanata@gmail.com.

\section{Информация об авторах}

Екатерина Владимировна Степанова, кандидат филологических наук, доцент кафедры лингвистики и межкультурной коммуникации, Волгоградский институт управления - филиал Российской академии народного хозяйства и государственной службы при Президенте РФ, г. Волгоград, Российская Федерация, ул. Гагарина, 8, 400131 г. Волгоград, Российская Федерация, stepanova.volg@mail.ru.

Наталья Евгеньевна Булетова, кандидат экономических наук, доцент, доцент кафедры государственного управления и политологии, Волгоградский институт управления - филиал Российской академии народного хозяйства и государственной службы при Президенте РФ, г. Волгоград, Российская Федерация, ул. Гагарина, 8, 400131 г. Волгоград, Российская Федерация, buletovanata@gmail.com.

\section{Informations sur les Auteurs}

Ekaterina V. Stepanova, Candidat des sciences philologiques, Professeur associé de la Chaire de Linguistique et de Communication Interculturelle de l'Institut de l'Administration Publique de Volgograd filiale de l'Académie Russe de l'Economie Nationale et de l'Administration Publique, 8, rue Gagarin, 400131, la ville de Volgograd, Russie, grajd@vags.ru.

Natalia E. Buletova, Candidat des sciences economiques, Professeur associé de la Chaire de l'administration publique et des sciences politiques de l'Institut de l'Administration Publique de Volgograd filiale de l'Académie Russe de l'Economie Nationale et de l'Administration Publique, 8, rue Gagarin, 400131, la ville de Volgograd, Russie, grajd@vags.ru. 